\title{
5 Warten auf den Innovationssprung - Warum („noch mehr“) Digitalisierung die ambulanten Leistungserbringer derzeit kaum begeistert
}

\author{
Andreas Rühle
}

Es gibt sie auch bei Leistungserbringern im Gesundheitswesen, die innovativen, digitalisierungsaffinen, stets auf Neuerung bedachten Early Adopters, die erkannt haben, dass die Gesundheitsversorgung der Zukunft Vernetzung benötigt. Aber sie sind sehr selten. Die „digitalisierte Welt“ der Leistungserbringer ist eine, die sich weitgehend in einem abgeschotteten, sektorinternen Umfeld bewegt, ohne Verbindung zu anderen Partnern. Dies betrifft nicht nur die Verbindung der verschiedenen Sektoren miteinander, z.B. Pflege und niedergelassene Ärzteschaft, dies betrifft auch die Leistungserbringer innerhalb eines Sektors, z.B. die digitale Kommunikation von Hausärzten mit Fachärzten.

Warum ist das so, und warum kann eine „digitale Vernetzungsmüdigkeit“ diagnostiziert werden? Was ist zu tun, um diese zu überwinden?

Innovationen in ein bestehendes System zu bringen ist generell keine einfache Aufgabe. Grundvoraussetzung ist neben Innovationsbereitschaft der Anwender das Einlösen der gegebenen Vorteilsversprechen. Für die direkt am Patienten tätigen Leistungserbringer im Gesundheitssystem kann sicherlich eine gewisse Reserviertheit gegenüber den verschiedenen digitalen Neuerungen konstatiert werden. Diese kommt aber nicht von ungefähr, sondern ist auch eine Folge von Unzulänglichkeiten einer Branche.

Wirft man einen Blick hinter die Kulissen der meisten ambulanten Leistungserbringer im Gesundheitssystem, tritt schnell digitale Ernüchterung ein: Die intersektorale Versorgungsrealität ist nur gering digital. 
5 Warten auf den Innovationssprung - Warum („noch mehr“) Digitalisierung

die ambulanten Leistungserbringer derzeit kaum begeistert

\section{Die nur begrenzt digitale Arztwelt}

Dass Praxisverwaltungssysteme in Arztpraxen Einzug erhalten haben und Standard sind, wird niemand ernsthaft bestreiten. Die Nutzung der Möglichkeiten eingesetzter Systeme zur Vereinfachung und Beschleunigung der Arbeitsprozesse ist aber oftmals auf einen Bruchteil dessen beschränkt was möglich ist. Die Chancen werden nicht erkannt, oder die Mitarbeiter sind nicht in der Lage, die Systeme über das hinaus zu bedienen, was zur Führung der elektronischen Karteikarte und zur Abrechnung erforderlich ist. Produktivitäts- und Informationsfortschritte sind durch die PVS-Systeme in der Praxis zwar spürbar; es wird dokumentiert, codiert, die Abrechnung elektronisch erstellt und übermittelt und an allen Arbeitsplätzen sind die relevanten Informationen verfügbar. Die Grenzen des Produktivitätsfortschrittes liegen aber an der Praxistür: Der Austausch mit anderen Fachgruppen findet in der Form statt, dass eingehende Briefe in Karteikarten einsortiert und bestenfalls eingescannt werden (mit Ausnahme der Übertragung und Integration von Labordaten).

Seit einigen Jahren haben sich viele kleine und große Unternehmen, aber auch Startups aufgemacht, digitale Vernetzung in die Arztpraxen bringen zu wollen. Themen wie gemeinsame Patientenakten, digitaler Austausch von Terminen, oder von Diagnose-, Therapie-, Medikamentendaten zwischen Haus- und Facharzt, digitale Übertragung von Bildern etc. werden immer wieder in vermeintlich bestens funktionierende Lösungen verpackt und vertrieben. In anderen Berufsfeldern zum Mindeststandard der Kommunikation und intersektoralen Zusammenarbeit gehörende Vernetzungen treffen in der Arztpraxis auf eine Welt, in der die Kommunikation mittels Fax und Telefon (noch) Standard ist. Die überwiegende Zahl der niedergelassenen Ärztinnen und Ärzte kommuniziert im Praxisalltag nicht per Datenaustausch oder verfügt über eine (sichere) Internetverbindung. Der Mikrokosmos Arztpraxis weist nur eine geringe Bereitschaft für eine Öffnung nach außen auf.

Es kann aber hieraus nicht konstatiert werden, dass eine grundlegende Ablehnung gegen Vernetzung und Datenaustausch bei niedergelassenen Ärztinnen und Ärzten besteht. Die bestehende „Müdigkeit“ und die Skepsis ist im Wesentlichen auch die Folge einiger Fehlentwicklungen.

Elektronische Patientenakte: Die seit Jahren nicht wirklich vorankommende Einführung einer einheitlichen, umfassenden sowie einrichtungs- und sektorübergreifenden elektronischen Patientenakte (ePA) für das Gesundheitswesen und die damit entstandene Diskussion um die Sicherheit der Patientendaten hat das Vertrauen in dieses Medium innerhalb der Ärzteschaft erschüttert. Noch immer bestehen Bedenken des Deutschen Ärztetages. Hauptforderung ist, dass die elektronischen Patientenakten in der Hand von Ärzten und Patienten verbleiben.

„Bestrebungen der Krankenkassen, elektronische Patientenakten zu entwickeln und in ihrer Obhut und mit ihrem Zugriff zu verwalten, werden zurückgewiesen. Elektronische Patientenakten sind ein gemeinsames Instrument einer vertrauensvollen und konstruktiven Patient-Arzt-Beziehung." (Entschließung des 119. Deutschen Ärztetages vom 24. bis 27.5.2016 in Hamburg)

„Der 120. Deutsche Ärztetag 2017 fordert die Bundesärztekammer auf, eigene Überlegungen anzustellen, bei denen ePA in der Obhut der Ärzteschaft verbleiben." (Beschluss des 120. deutschen Ärztetages vom 23. bis 26.5.2017 in Freiburg) 
Die mit viel Emotion geführten Diskussionen und die sich abzeichnende Kostenbelastung für Arztpraxen erhöhen den Widerstand gegen die Einführung.

E-Arztbrief: der elektronische Versand von Arztbriefen zwischen Fach- und Hausärzten sowie von Krankenhäusern wurde von der KVtelematik bereits im Jahr 2015 im Rahmen eines Pilottests mit ausgewählten Ärztenetzen initiiert. Voraussetzung war der - meist kostenpflichtige - Erwerb von Zusatzmodulen für die Praxisverwaltungssoftware und die Einrichtung eines KV-connect-Anschlusses. Der Anreiz für Krankenhäuser zur Teilnahme wurde in der im Entwurf zum E-Health-Gesetz stehenden Vergütung auch für Krankenhäuser gesehen. Im verabschiedeten E-Health-Gesetz war die Vergütung für Krankenhäuser gestrichen und die Vergütung für niedergelassene Ärzte gebunden an die Signatur mittels elektronischem Heilberufeausweis. Dies schreckte sowohl Krankenhäuser als auch Ärzte „im letzten Moment"ab. Praxen, die bereits in den E-Arztbrief investiert hatten waren vor den Kopf gestoßen.

\begin{abstract}
Vertrauensverlust digital: Seit vielen Jahren preisen Hersteller sogenannter elektronischer Vernetzungsmöglichkeiten in Präsentationen und Gesprächen ihre jeweilige Software und locken mit schnellerer, besserer und produktiverer Arbeitsweise. Doch die Realität ist ernüchternd: Nichts bis wenig funktioniert, ist einfach schlecht aufgesetzt oder scheitert an den Grenzen der Versorgungssysteme, da die Schnittstellen nicht bedient werden können. Von Vollintegration ganz zu schweigen.
\end{abstract}

Aber auch Softwareanbieter haben nicht zur Vertrauensbildung in diesem Sektor beigetragen. Immer wieder wurden Vernetzungslösungen angeboten, die eine Kommunikation zwischen verschiedenen Praxisverwaltungssystemen angeblich können sollten. Imaginäre Anwenderzahlen und erfolgreiche Installationen wurden genannt. Leider lösten sich fast alle Versprechen in Luft auf oder die Kommunikation war nur den Anwendern möglich, die die gleiche Software einsetzten. Ärzte und Arztnetze, die investiert hatten, waren die Verlierer. Bis heute werden die Schnittstellen gehütet wie der heilige Gral, von einer (möglichen) Anbindung von Krankenhäusern ganz zu schweigen.

Viele weitere Beispiele lassen sich finden. Am Ende blieben nicht funktionierende Vernetzungsversprechen und deren Kosten sowie ein großer Vertrauensverlust der potenziellen Anwender, die von der Sinnhaftigkeit und den Vorteilen eigentlich überzeugt sind bzw. waren.

\title{
Die nur begrenzt digitale Welt der Pflege und Anderer
}

Ähnlich sieht die Situation im Bereich der ambulanten und stationären Pflege aus: Auch hier erfolgt die Dokumentation in spezialisierten Softwaresystemen, die nicht mit Systemen anderer Sektoren kommunizieren. Insofern handelt es sich um ein Spiegelbild der Situation im ärztlichen Sektor. Ergänzt wird dies aber durch noch viel stärker ausgeprägte struktur- und ausbildungsbedingte Defizite im Umgang mit den Systemen. Gerade in ambulanten Pflegediensten gibt es oftmals immer noch nur 
5 Warten auf den Innovationssprung - Warum („noch mehr“) Digitalisierung

die ambulanten Leistungserbringer derzeit kaum begeistert

einen PC an dem die gesamte Dokumentation der Patientenversorgung durchgeführt wird. Die bestehenden Möglichkeiten einer Dokumentation „vor Ort“ nach dem Einsatz beim Patienten mittels Tablett oder Laptop werden zu wenig bis gar nicht genutzt. Meistens kommen die Mitarbeiter von der Patientenpflege ins Büro und dokumentieren oder lassen ihre manuellen Aufzeichnungen dokumentieren, da sie im Umgang mit den Systemen nicht geschult sind. Die Folge ist nicht nur ein zeitversetzter Eintrag sondern auch ein daraus resultierendes Informationsdefizit, da nicht jeder in die Pflege eines Patienten eingebundene Mitarbeiter zeitnah auf die relevanten Informationen zugreifen kann.

Hinzu kommt, dass der finanzielle Spielraum für erforderliche Investitionen in DVInfrastruktur im Sektor Pflege aufgrund der begrenzten Refinanzierbarkeit über Produktivitätsvorteile im Rahmen der Leistungserbringung am Patienten deutlich begrenzt ist.

Gleiches kann auch für andere Gruppen von Leistungserbringern konstatiert werden, etwa für Physiotherapeuten, Logopäden und ähnliche Berufsgruppen. Deren Dokumentation und Terminplanung erfolgt oft mittels tradierter Terminbücher und Karteikarten.

\section{Überwindung der digitalen Starre}

Eine mangelnde Bereitschaft zur Etablierung und Nutzung digitaler Vernetzungsvorteile kann den Leistungserbringern im Gesundheitswesen nicht unterstellt werden. Die bestehenden Vorbehalte gilt es zu überwinden, soll die digitale Vernetzung und Zukunft im Gesundheitswesen greifen. Hierzu sind folgende Punkte besonders wichtig:

- Rückgewinnung von Vertrauen: Durch das Nichteinhalten von Versprechen von Funktionalität, Produktivität und Informationsgewinn, die den interessierten Leistungserbringern gegeben wurden, ist viel Vertrauen verloren gegangen. Der Eindruck, dass unausgereifte Systeme verkauft wurden und werden, oder dass Lösungen implementiert werden, die an die Grundfragen des Versorgungsselbstverständnisses gehen, ist vorherrschend. Die Innovativen und für Vernetzung und Digitalisierung Aufgeschlossenen sind verprellt. Vertrauen kann nur zurückgewonnen werden durch nachweisbare Erfolge der angebotenen Lösungen sowie einen klaren Fahrplan für die Umsetzung, einschließlich Kostentransparenz und -verlässlichkeit.

- Erleben statt Versprechen von Vorteilen digitaler Vernetzung: Die Vorteile, die mit der Digitalisierung und Vernetzung verbunden sind, müssen klar und intersubjektiv objektivierbar sein. Dies bedeutet das Erleben im Rahmen von Probeinstallationen mit Echtbetriebssimulation. Echte Referenzeinführungen und -betriebe müssen benannt und besucht werden können. Das, was mit den jeweiligen Lösungen erreicht werden kann, was nicht, aber auch, was an Vorleistungen und Nachleistungen sowie Qualifizierung notwendig ist, muss im Vorfeld benannt werden. Die finanziellen Konsequenzen im Sinne vollkostenorientierten Lebenszykluskostenbetrachtung müssen aufgezeigt werden. Diese Transparenz ist Grundvoraussetzung für das Vermeiden von Unzufriedenheit oder gar einem Abbruch. 
- Kleine Schritte statt große Sprünge: Die Gefahr eines großen Wurfs ist immer die, dass ein unbefriedigendes Ergebnis erzielt wird. Das große Ziel wird in Angriff genommen, ohne dass die Voraussetzungen, die jeder einzelne Teilnehmer mitbringt, beachtet werden. Gerade bei Vernetzungen ist eine intensive Auseinandersetzung mit der jeweiligen (Ausgangs-)Situation der Teilnehmer geboten. Es müssen Teilziele definiert werden im Sinne von Etappenzielen. Jedes Etappenziel bedeutet aber auch Ausruhen für die nächste Wegstrecke, auch damit das Erreichte sich verfestigen kann.

- Implementierung: Begleitung und Hotline: Wichtig bei jeder Produkteinführung ist eine enge Begleitung bei der Implementierung. Dies betrifft nicht nur die Erfassung der systemseitigen Ist-Situation und Aufstellung der erforderlichen investiven Maßnahmen, z.B. in die EDV-Infrastruktur, sondern auch die Erfassung der individuellen Fähigkeiten derer, die später mit den Systemen arbeiten müssen. Hieraus muss ein differenziertes Schulungsangebot entstehen. Zu einer nachhaltigen Begleitung gehört auch eine Hotline für aktuelle Fragen und technischen Support. Die oftmals vorherrschende Praxis, zuerst eine Kostenvereinbarung über die Nutzung der Hotlines oder des technischen Supports zu schließen, schreckt ab. Erfolgreiche Anbieter haben einen Support ohne Zusatzkosten für den Nutzer. Dies ist leider nicht Standard, hilft aber, eine Einführung zügig und mit gutem Wissen um die Funktionalitäten sicherzustellen.

\section{Fazit}

Die vergangenen und derzeitigen Digitalisierungsanstrengungen gehen oftmals an der Versorgungsrealität und den Vorstellungen und Bedürfnissen der Leistungserbringer vorbei. Nur durch die intensive Auseinandersetzung mit dieser Realität kann eine Digitalisierung im Gesundheitswesen zukünftig nachhaltig von Erfolg geprägt sein. Dies erfordert Ehrlichkeit, funktionierende Lösungen und einen „langen Atem“ bei der Implementierung. An der grundlegenden Bereitschaft der Leistungserbringer für funktionierende Vernetzung sollte man nicht zweifeln. 